\title{
Assembly of ordered carbon shells on GaN nanowires
}

Eli Sutter, Peter Sutter, Raffaella Calarco, Toma Stoica, and Ralph Meijers

Citation: Appl. Phys. Lett. 90, 093118 (2007); doi: 10.1063/1.2710189

View online: https://doi.org/10.1063/1.2710189

View Table of Contents: http://aip.scitation.org/toc/apl/90/9

Published by the American Institute of Physics

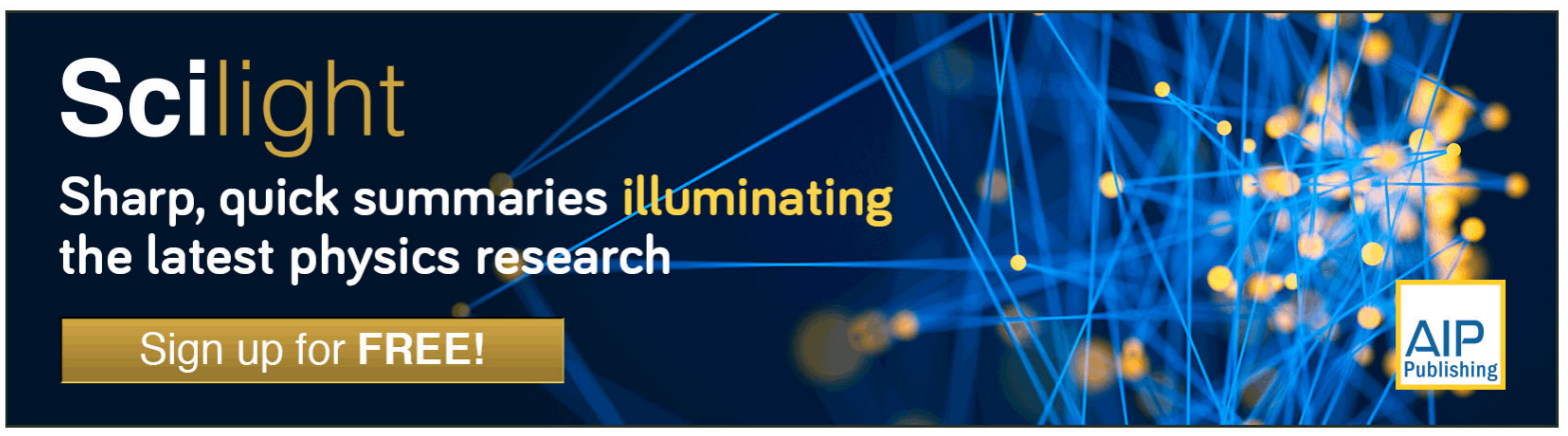




\title{
Assembly of ordered carbon shells on GaN nanowires
}

\author{
Eli Sutter ${ }^{\mathrm{a})}$ and Peter Sutter \\ Center for Functional Nanomaterials, Brookhaven National Laboratory, Upton, New York 11973
}

Raffaella Calarco, Toma Stoica, and Ralph Meijers

Institute for Bio- and Nano-Systems, and Center of Nanoelectronic Systems for Information Technology, Research Center Jülich, D-52425 Jülich, Germany

(Received 11 January 2007; accepted 25 January 2007; published online 1 March 2007)

\begin{abstract}
In situ annealing experiments on individual group III-nitride nanowires (NWs) in a transmission electron microscope demonstrate the encapsulation of GaN wires in crystalline carbon shells in the presence of $\mathrm{C}$ at moderate temperatures. The complete encapsulation of $\mathrm{GaN}$ wires in carbon shells can be achieved when small indium metal clusters are introduced on the wire surface. No encapsulation is observed on pure $\mathrm{GaN}$ wires under the same conditions. The observations suggest a general processing route for the formation of semiconductor core/C-shell NW structures based on surface decoration with small metal clusters. (C) 2007 American Institute of Physics.
\end{abstract}

[DOI: 10.1063/1.2710189]

Semiconductor nanowires (NWs) and more complex core-shell NW structures, in which a NW core is surrounded by a shell of a dissimilar material, ${ }^{1-5}$ have attracted a lot of interest recently as they are promising materials for a variety of quantum devices with functionality defined, for instance, by carrier confinement in the thin wires. Given the large surface-to-volume ratio of NWs and the strong effect of surface states on the electronic properties of a semiconductor (see, for instance, Refs. 6-8), the surface termination becomes especially important. At the very least, the free surface should be sufficiently stabilized and protected to enable handling in air without uncontrolled property changes. Multilayer carbon shells of nested graphene sheets, which are chemically inert, could provide such surface stabilization and passivation. ${ }^{9}$ Graphene shells around semiconductor NWs can also be used to establish core-shell nanostructures whose geometry - a metal/semimetal shell surrounding a semiconductor core-gives rise to a large-area Schottky junction. Such junctions could form the basis for innovative electronic and optoelectronic devices (e.g., photovoltaic cells), or could be used as sensing elements, drawing from an already comprehensive understanding of the functionalization of carbon surfaces.

In contrast to nanoparticles of some metals that are readily encapsulated in graphitic shells in the presence of sufficient carbon, ${ }^{10}$ the surface chemistry of semiconductors precludes the growth of ordered $\mathrm{C}$ sheets. We have recently demonstrated that the absence of catalytic effects in semiconductors can be circumvented and $\mathrm{C}$ shells can be formed around nanowires whose surface is decorated with small amounts of metal. ${ }^{9}$ Real-time observations by highresolution transmission electron microscopy (HRTEM) of the controlled encapsulation of Ge NWs in ordered C shells during annealing of individual carbon-supported $\mathrm{Ge}$ nanowires showed that the assembly of the $\mathrm{C}$ shells depends critically on the presence of small amounts of metal on the NW surface. In the case of Ge NWs, the metal was residual $\mathrm{Au}$ in the form of small clusters on the wire surface originating from

\footnotetext{
${ }^{\text {a) }}$ Author to whom correspondence should be addressed; Fax: 631-344-3093;
} electronic mail: esutter@bnl.gov the $\mathrm{Au} / \mathrm{Ge}$ catalyst nanoparticles used for the wire synthesis. ${ }^{9}$ Here we use experiments on group III-nitrides, a class of NW materials particularly important for optoelectronic applications, to generalize the approach of using surface metal decoration to trigger the encapsulation of semiconductor NWs in C shells. Via in situ TEM experiments we demonstrate the controlled encapsulation of individual GaN wires in carbon shells during annealing at moderate temperatures. While we find it impossible to encapsulate pure $\mathrm{GaN}$ NWs, intentionally introduced small amounts of In at the NW surface trigger the efficient assembly of fragments of graphene $\mathrm{C}$ sheets, which then grow into well-defined multilayer $\mathrm{C}$ shells that completely encapsulate the $\mathrm{GaN}$ NWs. While a somewhat similar encapsulation of $\mathrm{GaN}$ in carbon nanotubes has been demonstrated in arc-discharge and microwave-plasma-enhanced chemical vapor depositions assisted by $\mathrm{Ni}$ and $\mathrm{Fe}$ catalysts, respectively, ${ }^{11,12}$ the use of transition metal catalysts inevitably results in undesired contamination $^{13}$ and degradation of the electronic properties of the NWs. ${ }^{14}$

Our variable temperature in situ experiments were carried out in a JEOL JEM 3000F field-emission TEM equipped with a Gatan 652 high-temperature sample holder. They covered the temperature range between room temperature and $500{ }^{\circ} \mathrm{C}$ at pressures of $2 \times 10^{-5} \mathrm{~Pa}$. The NWs were fabricated prior to the in situ studies by radio frequency plasmaassisted molecular beam epitaxy without the use of a catalyst. GaN NWs were grown on $\mathrm{Si}$ (111) substrates at $790{ }^{\circ} \mathrm{C}$ in an UHV chamber with a base pressure of $2 \times 10^{-10}$ torr. $^{15}$ Indium-decorated GaN NWs were realized by an additional deposition of $\sim 15 \mathrm{~nm}$ of InGaN with a nominal In:Ga ratio of 2.1:3 under the same conditions as the GaN growth. Under these conditions well-defined InGaN layers were not observed, but In clusters on the NW surfaces formed instead, consistent with earlier observations. ${ }^{16}$ These NW materials were transferred through air and dispersed on amorphous carbon films, which act as a carbon reservoir for the variable temperature experiments.

Figures 1(a)-1(d) show the structure and morphology of GaN NWs representative of the as-grown starting material of our study. The GaN NWs attached to the Si substrate are 

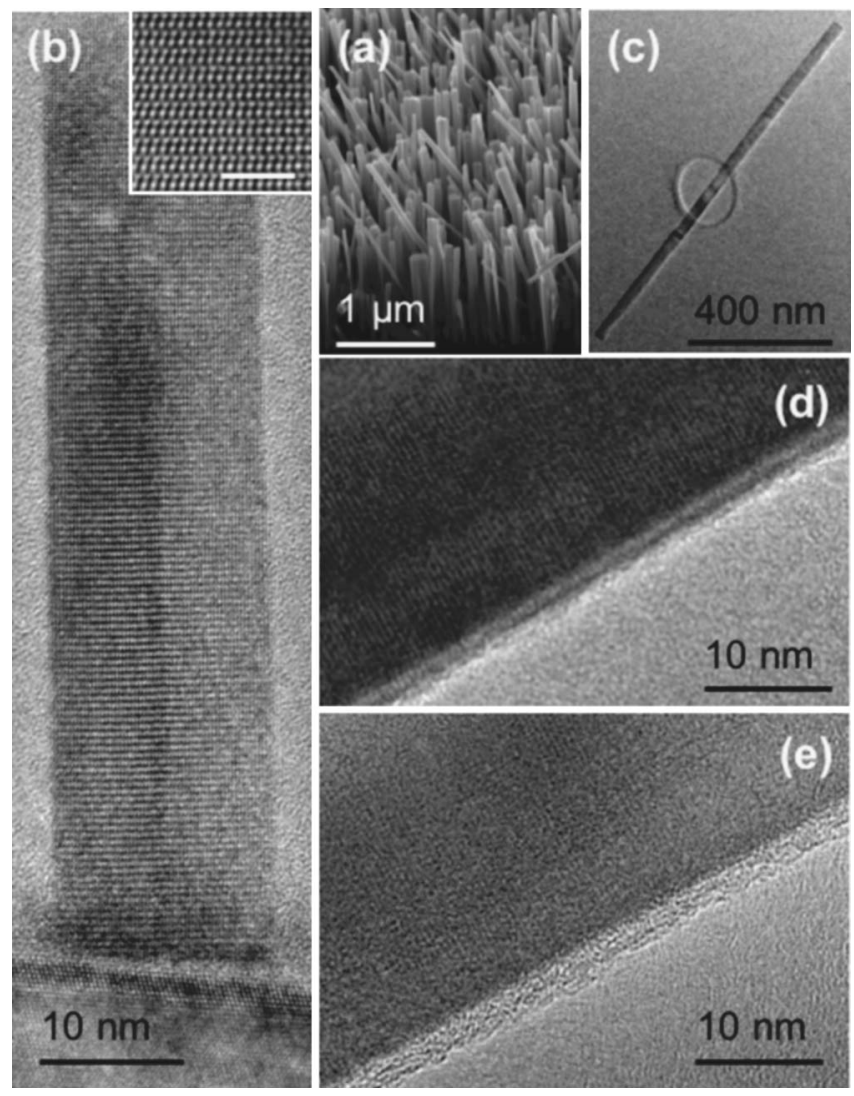

FIG. 1. (a) Overview SEM and (b) detail high-resolution TEM images of catalyst-free GaN NWs on $\mathrm{Si}(111)$ substrate. The inset in (a) shows a detail of the crystal structure (scale bar of $1 \mathrm{~nm}$ ). (c) One of the GaN NWs dispersed on the amorphous carbon film for the in situ annealing experiments. (d) TEM image of the area close to the surface of the GaN NW from (c). (e) Same section of the NW after in situ annealing at various temperatures between $\mathrm{RT}$ and $500{ }^{\circ} \mathrm{C}$.

shown in the scanning electron microscope image in Fig. 1(a) and in the cross-sectional HRTEM image of Fig. 1(b). The NWs are monocrystalline wurtzite GaN with growth direction along the $c$ axis. They have well-defined faceted side and front surfaces and there is no obvious metal-rich tip, in contrast to NWs grown on metal catalyst nanoparticles. The wires are straight, with a typical diameter of $10-50 \mathrm{~nm}$ that is constant over the length of the NW, and a length of about $1 \mu \mathrm{m}$. The NWs dispersed on the amorphous carbon film for the in situ TEM experiments are shown in Fig. 1(c). The wire in the figure is $1.1 \mu \mathrm{m}$ long and has a diameter of $35 \mathrm{~nm}$. The TEM image in Fig. 1(d) shows the detailed structure of the wire close to its surface. Figure 1(e) shows the same section of the NW after in situ annealing in the TEM at various temperatures between $\mathrm{RT}$ and $500{ }^{\circ} \mathrm{C}$. The temperature was increased slowly to $500{ }^{\circ} \mathrm{C}$ during imaging, using conditions similar to those in a previous study under which Au-decorated Ge NWs were completely encapsulated in an ordered multilayer shell of graphitic carbon. ${ }^{9}$ In contrast, no assembly of ordered $\mathrm{C}$ sheets was observed on the $\mathrm{GaN}$ NWs, which showed only the buildup of an amorphous $\mathrm{C}$ layer [Fig. 1(e)]. This layer remained amorphous and did not undergo any ordering even after prolonged annealing up to $500{ }^{\circ} \mathrm{C}$ in the microscope. The GaN NW structure remained unaffected as expected at these moderate temperatures.

GaN NWs with small amounts of In on the surface demonstrated a very different behavior under the same experimental conditions. One such NW after in situ annealing is
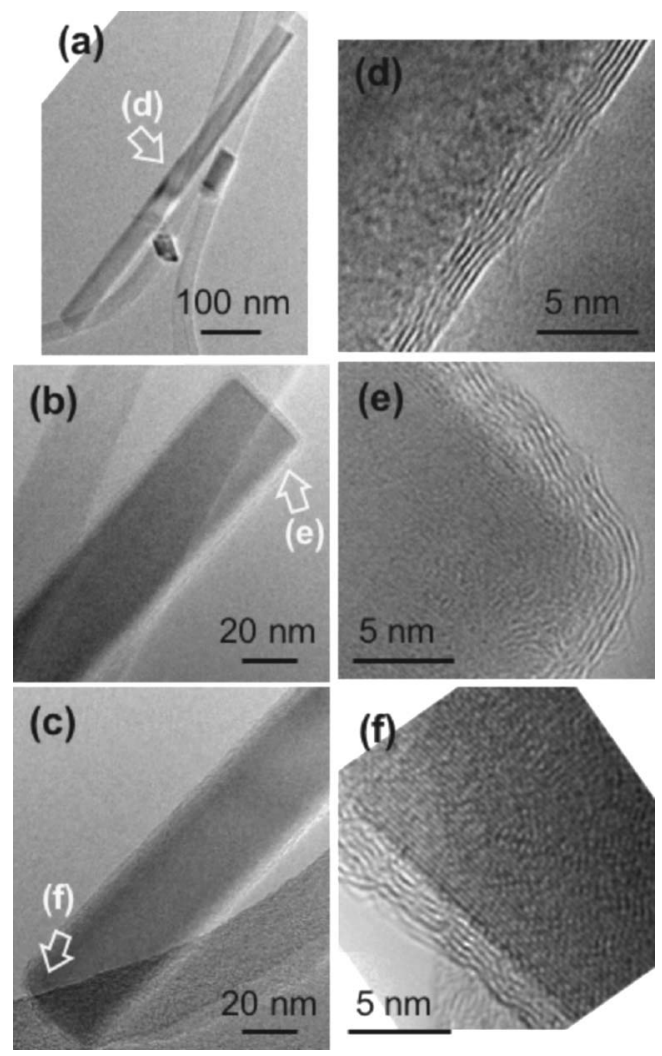

FIG. 2. TEM images showing a typical In-terminated GaN NW completely embedded in a $\mathrm{C}$ shell. (a) Overview image showing the entire NW. [(b) and (c)] Higher magnification images of two sections of the NW showing the GaN core and the $\mathrm{C}$ shell. [(d) and (f)] images of the $\mathrm{C}$ shell taken at different positions along the NW indicated by arrows in (a)-(c).

shown in Fig. 2(a). Higher magnification TEM images of sections near the two ends of the NW [Figs. 2(b) and 2(c)] show that an ordered graphene $\mathrm{C}$ shell continuously covers the entire NW surface. Details of the shell are shown in high-resolution images in Figs. 2(d)-2(f), obtained on different parts of the NW indicated by arrows in Figs. 2(a)-2(c). The shell is uniform in thickness over the entire surface of the NW and consists of about five graphene layers with a measured spacing of $0.35-0.36 \mathrm{~nm}$, which agrees well with the $c$ axis spacing of graphene sheets in graphite.

Unlike the GaN NWs in Fig. 1, which show homogeneous TEM contrast throughout, both before and after the in situ annealing experiments, the contrast of the In-terminated NWs is heterogeneous with significant variations on the atomic scale. Small spots exhibiting darker contrast can be clearly seen [Figs. 2(d)-2(f) and Fig. 3]. We attribute these spots to In clusters randomly dispersed on the $\mathrm{GaN}$ surface. From the TEM images the size of the In clusters appears to be less than $1 \mathrm{~nm}$ and they do not show any crystalline structure. A closer look at the NW/C-shell interface shows that the first layers of the shell invariably consist of small curved graphene fragments that envelope In clusters. Away from the interface, these initial graphene fragments are coated by progressively larger sheets that gradually become less curved and finally develop into extended, continuous graphene layers at the surface of the shell (Fig. 3).

Our finding that In terminated GaN NWs are readily encapsulated in ordered $\mathrm{C}$ shells under conditions that preclude the assembly of graphene shells around pure GaN NWs suggests that the observed small In clusters at the NW 

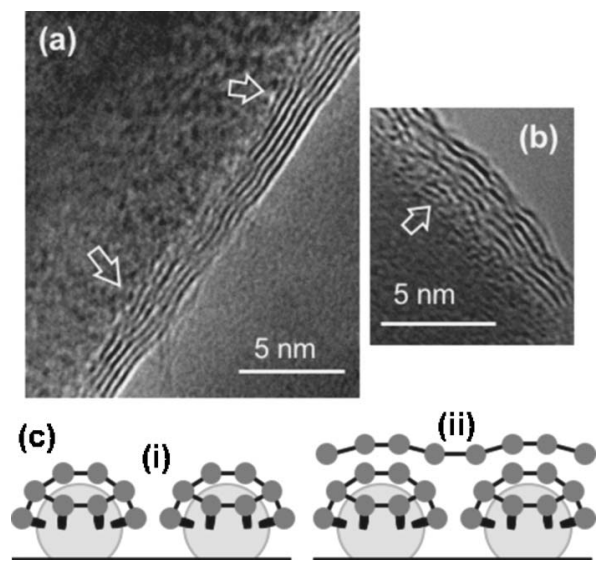

FIG. 3. [(a) and (b)] TEM images of the core/shell interface of an Indecorated GaN NW embedded in a $\mathrm{C}$ shell. The arrows mark the position of some of the In clusters at the NW surface. (c) Schematic representation of the initial stages of the assembly of the carbon shell-envelope of curved graphene flakes around In clusters (i), serving as a template for extended graphene layers (ii).

surface are instrumental to the assembly of the multilayer $\mathrm{C}$ shells. Furthermore, the particular morphology of the initial $\mathrm{C}$ sheets, small graphene fragments attached to and wrapping around In clusters, indicates that the assembly and binding of crystalline carbon sheets require small metal clusters. Indeed, under the same conditions the in situ $\mathrm{C}$ exposure of more extended In surfaces, $2-10 \mathrm{~nm}$ thick In layers obtained by the decomposition of the surface of pure InN NWs (at rates agreeing well with literature values ${ }^{17}$ ) during prolonged annealing at $\sim 450{ }^{\circ} \mathrm{C}$, did not result in the assembly of an ordered C shell.

Stoichiometric GaN is stable at the moderate annealing temperatures of our experiments. The nitride does not react with carbon, ${ }^{11}$ and its surface chemistry and reactivity preclude the growth of ordered $\mathrm{C}$ sheets, which, as demonstrated in the present experiments with In and in our previous study using $\mathrm{Au},{ }^{9}$ requires the catalytic effects of a small metal cluster. Once the NW surface is decorated with In clusters, the behavior changes drastically and the nanometersized graphene sheets readily assemble from the available $\mathrm{C}$ atoms. The fact that extended In surfaces do not have this effect suggests that the abundant low-coordination sites and the overall surface curvature of small metal clusters play a key role in the assembly of the initial graphene sheets. Simulations of metal $(\mathrm{Ni}, \mathrm{Fe})$ catalyzed growth of single wall $\mathrm{C}$ nanotubes have shown that, in addition to an available supply of carbon, two conditions have to be fulfilled for nanotube growth to occur ${ }^{18}$ (i) the strong covalent bonding of single $\mathrm{C}$ atoms to the catalyst and (ii) the firm adhesion of $s p^{2}$ bonded $\mathrm{C}$ in small graphene fragments to the metal surface. Neither In considered here nor Au used in our previous study ${ }^{9}$ is known to be good catalyst for nanotube growth, likely because their weak binding of $\mathrm{C}$ adatoms and relatively weak interaction with graphene sheets preclude long term anchoring and growth by root incorporation of C. ${ }^{18}$ However, our experiments show that small supported clusters of these metals interact sufficiently with $\mathrm{C}$ atoms and $s p^{2}$ $\mathrm{C}$ assemblies, likely via an overlap of C-derived states and metal $d$ states near the Fermi energy, ${ }^{19,20}$ to act as templates for the growth of curved graphene flakes, which in turn can be overgrown by additional sheets to generate a multilayer $\mathrm{C}$ shell. ${ }^{21}$ Importantly, the assembly of the $\mathrm{C}$ shell and encapsulation of GaN NWs by decoration of the wire with group III (In) metal clusters avoid the usual transition metal catalysts, whose incorporation as trace impurities would give rise to defect states deep in the band gap and would consequently degrade the electronic and optoelectronic properties of GaN. ${ }^{14}$ Our strategy can therefore be expected to produce $\mathrm{GaN} / \mathrm{C}$ core-shell structures that preserve the intrinsic electronic properties of the nitride core.

In conclusion we have demonstrated the formation of ordered $\mathrm{C}$ shells around GaN NWs at moderate annealing temperatures that do not affect the crystallinity of the wires. The formation of the shells was achieved by decorating the surface of the NWs with small In clusters that serve as an initial template for the $\mathrm{C}$ encapsulation. More generally our findings demonstrate that a similar encapsulation in a graphene $\mathrm{C}$ shell can be achieved on any semiconductor NW material provided that the surface of the semiconductor is decorated with small metal clusters.

This work was performed under the auspices of the U.S. Department of Energy, under Contract No. DE-AC0298CH1-886.

${ }^{1}$ J. Xiang, W. Lu, Y. Hu, Y. Wu, H. Wan, and C. M. Lieber, Nature (London) 441, 489 (2006).

${ }^{2}$ F. Qian, Y. Li, S. Gradecak, D. Wang, C. J. Barrelet, and C. M. Lieber, Nano Lett. 4, 1975 (2004).

${ }^{3}$ N. Sköld, L. S. Karlsson, M. W. Larsson, M.-E. Pistol, W. Seifert, J. Traghardh, and L. Samuelson, Nano Lett. 5, 1943 (2005).

${ }^{4}$ W. Lu, J. Xiang, B. P. Timko, Y. Wu, and C. M. Lieber, Proc. Natl. Acad. Sci. U.S.A. 102, 10046 (2005).

${ }^{5}$ T. Bryllert, L. E. Wernersson, T. Lowgren, and L. Samuelsson, Nanotechnology 17, S227 (2006).

${ }^{6}$ R. Calarco, M. Marso, T. Richter, A. Aykanat, R. Meijers, A. V. D. Hart, T. Stoica, and H. Lüth, Nano Lett. 5, 981 (2005).

${ }^{7}$ T. Stoica, R. Meijers, R. Calarco, T. Richter, E. Sutter, and H. Lüth, Nano Lett. 6, 1541 (2006).

${ }^{8}$ T. Hanrath and B. Korgel, J. Phys. Chem. B 109, 5518 (2005); D. Wang, Y.-L. Chan, Z. Liu, and H. Dai, J. Am. Chem. Soc. 127, 11871 (2005); V. Schmidt, S. Senz, and U. Gösele, Appl. Phys. A: Mater. Sci. Process. 86, 187 (2007).

${ }^{9}$ E. Sutter and P. Sutter, Adv. Mater. (Weinheim, Ger.) 18, 2583 (2006).

${ }^{10}$ E. Sutter, P. Sutter, and Y. Zhu, Nano Lett. 5, 2092 (2005); F. Banhart, Rep. Prog. Phys. 62, 1181 (1999).

${ }^{11}$ W. Han and A. Zettl, Adv. Mater. (Weinheim, Ger.) 14, 1560 (2002).

${ }^{12}$ C. Y. Zhi, D. Y. Zhong, and E. G. Wang, Chem. Phys. Lett. 381, 715 (2003).

${ }^{13}$ E. Stach, P. Pauzauskie, T. Kuykendall, J. Goldberger, R. He, and P. Yang, Nano Lett. 3, 867 (2003).

${ }^{14}$ J. A. Chisholm and P. D. Bristowe, Modell. Simul. Mater. Sci. Eng. 9, 249 (2001).

${ }^{15}$ R. Meijers, T. Richter, R. Calarco, T. Stoica, H.-P. Bochem, M. Marso, and H. Lüth, J. Cryst. Growth 289, 381 (2006).

${ }^{16}$ C. Adelmann, R. Langer, G. Feuillet, and B. Daudin, Appl. Phys. Lett. 75, 3518 (1999).

${ }^{17}$ Y. Huang, W. Wang, Q. Sun, J. Chen, J. F. Wang, Y. T. Wang, and H. Yang, J. Cryst. Growth 281, 310 (2005).

${ }^{18}$ J.-Y. Raty, F. Gygi, and G. Galli, Phys. Rev. Lett. 95, 096103 (2005).

${ }^{19}$ S. Helveg, C. Lopez-Cartes, J. Sehested, P. L. Hansen, B. S. Clausen, J. R. Rostrup-Nielsen, F. Abild-Pedersen, and J. K. Norskov, Nature (London) 427, 426 (2004).

${ }^{20}$ J. Zhang, K. Sasaki, E. Sutter, and R. Adzic, Science 315, 220 (2007).

${ }^{21}$ The organization of the initial small graphene sheets into closed graphitic multilayers may be promoted by the electron beam in our experiments. See, for example, D. Ugarte, Carbon 33, 989 (1995). 